\title{
A Tribute to Mahir Saleh Hussein (1944-2019)
}

\author{
Carlos Bertulani $^{1} \cdot$ Arnaldo Gammal $^{2} \cdot$ Valdir Guimaraes $^{2}$
}

Published online: 5 January 2021

(C) Sociedade Brasileira de Física 2021

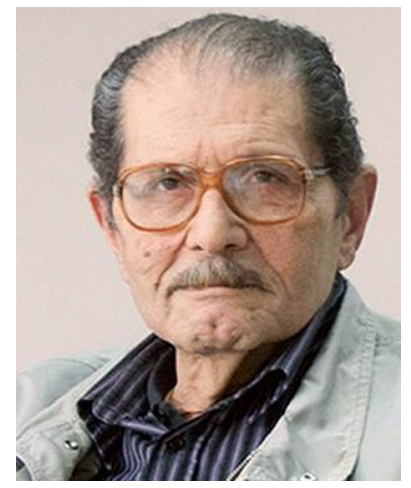

Mahir Saleh Hussein passed away on 16 May 2019 in Sao Paulo, Brazil. He had a long and distinguished career in nuclear reaction theory, physics of exotic nuclei, accelerator physics, quantum chaos and applications to nuclei and mesoscopic systems, and Bose-Einstein condensation.

Mahir was born on 21 November 1944 in Baghdad, Iraq, and proudly maintained his Iraqian citizenship throughout his life. He attended public schools in several districts of Baghdad and eventually entered the College of Science in the University of Baghdad (UB) during the period 1964-1965. He was the first graduate student of the UB in 1965. In 1967, he went to the Massachusetts Institute of Technology (MIT), where he was granted a Ph.D. in Physics in 1971 under the guidance of Prof. Arthur Kerman. Immediately after his graduation, he accepted a position on October 1971 at the University of Sao Paulo (USP), where he was among the most energetic scientists, working at the University for the next

Carlos Bertulani

carlos.bertulani@tamuc.edu

1 Texas A\&M University-Commerce, Commerce, TX 75428, USA

2 Universidade de Sao Paulo, Cidade Universitária, São Paulo 05508-090, Brazil

47 years. He became an associate professor in 1977 and a full professor in 1987. By the end of his career, he had published over 300 papers in refereed journals.

Mahir was a scientific entrepreneur with a close eye to the newest developments in physics and astronomy. He was very broad and a motivating person, always optimistic of his theoretical predictions. This allowed him to collaborate with the finest physicist around the world. He holds a distinguished Tinker Visiting Professor at the University of Wisconsin at Madison during the year 1979-1980 and a Smithsonian visiting professor at MIT in 1994-1995. He was also a visiting scientist at the Institute of Theoretical Atomic and Molecular Physics at Harvard University. He served as the Scientific Secretary of the DOE-NSF Nuclear Science Long Range Plan in 1995. At the time, a close friend, E. J. Moniz (MIT), was the Chair of NSAC. Mahir did not turn down important administrative tasks and served as the head of the Nuclear Physics Department of the Instituto de Física of the University of São Paulo from 1995 to 1999.

Despite being a theorist, Mahir was a person with a strong vision on the needs of experimental physics. During his term as department head, a decision was made to install a superconducting solenoid system for production of radioactive ion beams at the University. He then coordinated to obtain the first grant for the purchase of the solenoids and ancillary systems, a project which became known as RIBRAS, for Radioactive Ion Beams in Brazil. Later, while working on several other subjects, he never stopped helping his colleagues with the theoretical interpretation of results obtained with RIBRAS. This happened up to the last few months prior to his passing. The study of the reactions induced by radioactive nuclei in this laboratory shed light on several important issues in nuclear physics and astrophysics.

Over the last 25 years Mahir had been involved rather heavily in four major research efforts: (1) laser-driven accelerators, (2) Theoretical Nuclear Physics, (3) Quantum Chaos Theory and Applications, and (4) Theory of Bose-Einstein Condensation. Of the four, the second, Theoretical Nuclear Physics, was his main research specialty. He had, in 
collaboration with several colleagues, developed the reaction theory for exotic (neutron- or proton-rich) nuclei, a detailed model for the excitation and decay of multiple giant resonances and studied in details fundamental symmetry violation in nuclei. He had also collaborated with experimental colleagues in Brazil, USA, Germany, and France, in interpreting data in the field of heavy-ion reactions and dynamics as well as in assessing the degree of isospin violation in light nuclei. After his retirement in 2007 and till his passing, Mahir chaired the Non-Conventional Astrophysics group at the Advanced Studies Institute (IEA) of the USP where he organized yearly workshops, also on topics of Bose-Einstein condensation, quantum chaos, and cosmology. He also maintained till the end his activities at the Institute of Physics of the University of Sao Paulo.

Because they were so many, it is difficult to summarize the major awards and honors received by Mahir during his long and distinguished career. He was a J.S. Guggenheim Fellow in 1987-1988, doing research at the University of Wisconsin, Madison. He was a Smithsonian Foundation Fellow in 1995, spending his time at the MIT and working together with Herman Feshbach, Arthur Kerman, Ernest Moniz, and with
Franco Iachello (Yale) on nuclear reaction theory and the foundations of quantum mechanics. He was a Martin Gutzwiller Fellow at the Max-Planck Institute for Physics of Complex Systems in Dresden, 2007-2008, where he worked on quantum chaos and Bose-Einstein condensation. Mahir was a member of the Brazilian Academy of Sciences, of the World Academy of Sciences and was a Fellow of the American Physical Society. He was proud to mention that he had always been very active in helping Iraqi scientists to get involved in exchange programs. He was doing this through his association, as a member of the Board of Directors of the Iraqi Society for Higher Education Abroad.

As a tribute to Mahir, the Brazilian Journal of Physics invited some of Mahir's collaborators to contribute to a volume dedicated to his memory. This volume is a collection of independent works by the invited physicists. All articles have been peer-reviewed and judged by the progress they bring to the different areas of physics that Mahir was active.

Publisher's Note Springer Nature remains neutral with regard to jurisdictional claims in published maps and institutional affiliations. 